\title{
The evolution of time-intensity curves of contrast enhanced ultrasonography in early arthritis patients with wrist involvement.
}

\author{
Maria-Magdalena Tămaş¹, Cosmina Ioana Bondor², Nicolae Rednic ${ }^{3}$, Linda Jessica Ghib ${ }^{4}$, \\ Simona Rednic ${ }^{1}$
}

${ }^{1}$ Rheumatology Department, "Iuliu Haţieganu” University of Medicine and Pharmacy, ${ }^{2}$ Department of Informatics and Biostatistics, "Iuliu Haţieganu" University of Medicine and Pharmacy, ${ }^{3} 4^{\text {th }}$ Medical Department, Railway Hospital, ${ }^{4}$ Rheumatology Department, Clinical Emergency County Hospital, Cluj-Napoca, Romania

\begin{abstract}
Aims: The aim of the study was to assess the evolution of time-intensity curves parameters of contrast-enhanced ultrasonography (CEUS) after 6 months of conventional treatment in early arthritis patients with wrist involvement. Material and methods: Patients diagnosed with early rheumatoid arthritis or undifferentiated arthritis on the basis of $2010 \mathrm{ACR} / \mathrm{EU}-$ LAR classification criteria, with bilateral wrist arthritis and both radiocarpal (RC) and intercarpal (IC) synovial hypertrophy identified by grey-scale ultrasonography, were enrolled. Synovial hypertrophy was semi-quantitatively scored (grade 0 -3) by grey-scale and by Power Doppler at wrist level. CEUS was performed using Sonovue. The region of interest was selected as the area corresponding to the synovial hypertrophy of the RC and IC joints. Time-intensity curves parameters were calculated with Contrast Dynamic Software. The minimum and the maximum values of Peak, area under the curve (AUC), and slope were selected for each patient at baseline and after 6 months of conventional treatment. The difference between the visits was noted as " $\Delta$ ". Results: Eleven patients fulfilled the inclusion criteria. Maximum time-intensity curves parameters' difference significantly decreased at 6 months: Peak $(30.00 \pm 5.90 \%$ vs $23.22 \pm 5.22 \%, p=0.008)$, AUC $(1206.08 \pm 216.91 \%$ s vs $949.13 \pm 280.12 \% \mathrm{~s}, p=0.04)$ and slope $(1.6(1.4 ; 2.3) \% / \mathrm{s}$ vs $1(0.7 ; 1.2) \% / \mathrm{s}, p=0.03)$. Moderate correlations were found between maximum $\triangle$ Peak, maximum $\triangle \mathrm{AUC}$ and maximum $\triangle$ Power Doppler grade $(\mathrm{r}=0.44, \mathrm{p}=0.17 ; \mathrm{r}=0.46, \mathrm{p}=0.16$, respectively). Conclusions: Peak and AUC for joints that had high baseline values significantly decreased following treatment with conventional synthetic drugs in EA patients with wrist arthritis. This decrease in Peak and AUC was moderately correlated with a decrease in US parameters. The joint with the highest values of these parameters may be used for evaluation of EA patients at follow-up.
\end{abstract}

Keywords: contrast-enhanced ultrasonography, time-intensity curves, early arthritis

\section{Introduction}

There is increasing evidence for the need of quantification of inflammation in rheumatoid arthritis (RA) [14]. Ultrasonography (US) has proven its superiority over clinical examination in detecting synovial hypertrophy [5-7] and power Doppler US (PDUS) is currently used

Received 20.06.2015 Accepted 18.07.2015

Med Ultrason

2015, Vol. 17, No 3, 345-351

Corresponding author: Maria-Magdalena Tămaş

Department of Rheumatology

2-4 Clinicilor Street,

400006 Cluj-Napoca, Romania

Phone +40264591942/1678, Fax +40264-431040

E-mail: mariam.tamas@gmail.com for the semiquanitative grading of intraarticular inflammation.

Although clinical assessment is essential for the follow-up in RA patients, the use of clinical scores may not be the ideal instrument for measuring the disease activity, due to other factors that influence the pain or biological markers of inflammation. The lack of correlation between clinical and US assessments in patients in clinical remission has already been demonstrated $[8,9]$. The use of PDUS in the "treat-to-target" strategy of RA patients may improve the evolution [10], as PD was demonstrated to be a poor prognostic factor both in RA and early RA $[11,12]$.

Contrast enhanced ultrasonography (CEUS) allows a better visualization of blood flow in small vessels with 
low velocity, providing more information on synovial inflammation comparing to PDUS [13-17]. Objective CEUS measurements using time-intensity curves (TIC) parameters calculated by dedicated software may provide information about the disease activity in RA [18]. Previous studies have suggested a possible role of CEUS in therapy monitoring [19-23]. Using TIC parameters in the follow-up of RA patients would allow a more objective measurement of synovial inflammation.

However, a definite role of CEUS in daily rheumatologic clinical practice has not been assigned. Due to the short life of the contrast agent, CEUS can be performed only at one joint level at a time. There is no evidence yet regarding the joint being examined by CEUS in a polyarticular disease such as RA, nor which TIC parameters to use for assessing synovial inflammation. Platzgummer $\mathrm{H}$ et al [18] showed that the area under the curve (AUC) correlated with PD scores and Song IH et al [24], using the slope value from the TIC parameter, demonstrated a good correlation between CEUS and magnetic resonance imaging.

The main aim of our study was to assess the evolution of TIC parameters in early arthritis (EA) patients presenting wrist arthritis, treated with conventional synthetic drugs for 6 months. Secondly, we compared the response to treatment, as measured by TIC parameters, of joints with different CEUS activities at baseline, in order to observe possible correlations with clinical and US evolution. As a third objective, we investigated which of the TIC parameters is more suitable for evaluating EA.

\section{Material and methods}

Patients diagnosed with early RA (using the 2010 ACR/EULAR classification criteria [25]) or with early undifferentiated arthritis, presenting with bilateral wrist arthritis were enrolled. The study was performed in a tertiary department of Rheumatology between June-July 2014. Synovial hypertrophy of both radiocarpal (RC) and intercarpal (IC) joints, bilateral, had to be documented by grey scale US (GSUS), longitudinal scan over the third extensor tendon, before inclusion. Clinical assessment, US, and CEUS were performed at baseline and at 6 months follow-up visits.

The study was conducted according to the Declaration of Helsinki and local regulations. The institutional Ethics Committee of the "Iuliu Haţieganu" University of Medicine and Pharmacy approved the study and written informed consent was given by all patients.

\section{Study design}

Four joints - IC and RC, bilaterally - were examined by CEUS. US examinations were performed after each patient had rested for at least 30 minutes in a constant room temperature. The region of interest (ROI) for CEUS was selected as the area corresponding to the synovial hypertrophy of the IC and RC joint prior identified in grey scale US. Peak, AUC and slope were analyzed at baseline in each of the four examined joints. For each patient we selected the joints with maximum $(\max )$ and minimum $(\mathrm{min})$ values of each TIC parameter, obtaining experimental groups named "Max Joints" and "Min Joints", respectively, for each TIC parameter. These joints were subsequently analyzed at the follow-up visit and the difference between baseline and follow-up for each TIC parameter was noted as " $\Delta$ " (delta).

US and CEUS examinations were performed by the same experienced sonographer ( $>5$ years musculoskeletal US).

\section{Patients evaluation}

Twenty-eight joints included in the disease activity score (DAS28) were assessed for tenderness and swelling at baseline and at 6 months follow-up. Patient and physician global assessment of the disease activity (PGA and PhGA) reported on a $100 \mathrm{~mm}$ visual analogue scale, and the duration of morning stiffness were recorded. C reactive protein (CRP, normal levels $<0.6 \mathrm{mg} / \mathrm{dl}$ ), erythrocyte sedimentation rate $(\mathrm{ESR}$, normal $<20 \mathrm{~mm} / \mathrm{h}$ ) as well as IgM rheumatoid factor (RF, negative $<32 \mathrm{UI} / \mathrm{ml}$ ) and anti-CCP antibodies (negative $<5 \mathrm{UI} / \mathrm{ml}$ ) were determined. DAS28, clinical disease activity index (CDAI), and symplified disease activity index (SDAI) scores were calculated.

\section{Ultrasonography}

Equipment

US examinations were performed using $18 \mathrm{MHz}$ probe for grey scale US and PDUS and $9 \mathrm{MHz}$ probe for CEUS (Acuson S2000 machine, Siemens Healthcare). Cadence CPS Technology was used for CEUS technique. The analysis of TIC was performed with Contrast Dynamic Software (Siemens Healthcare).

\section{Grey-scale US and PDUS}

US examinations were performed in order to detect synovial inflammation using the techniques and definitions described for musculoskeletal US [26,27]. Both wrists were scanned from the dorsal aspect, to detect synovial inflammation. PD settings were optimized to low wall filters, a pulse repetition frequency of $750 \mathrm{~Hz}$ and the colour gain was set below noise floor. Plenty of gel was used and attention was paid by the examiner to avoid compression of the tissue [28]. Synovial hypertrophy was semiquantitatively analyzed ( 0 absent, 1 mild, 2 moderate, 3 severe), for each RC and IC joint and PDUS was semiquantitatively scored (grade 0-3) in the same joints, as described by Szkudlarek et al [29]. 


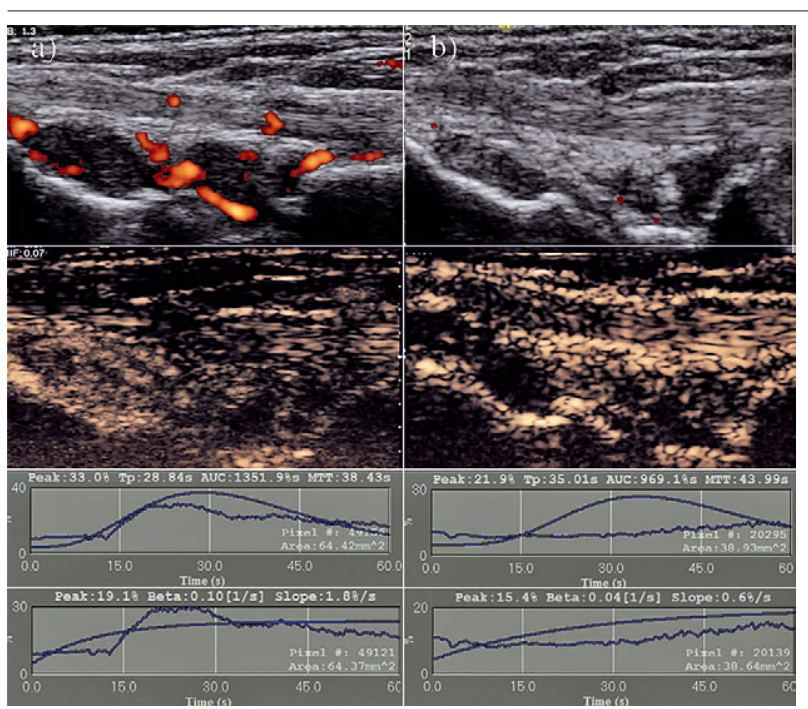

Fig 1. Right wrist joints in a patient with early RA at baseline (a) and at follow-up (b): PDUS for IC and RC joints; CEUS enhancement of the IC joint at the maximum intensity; TIC parameters of the IC joint in gamma and exponential analysis.

\section{CEUS}

The settings included a low mechanical index (0.07) and 60 seconds per cine video-clip. For each examination $2.4 \mathrm{ml}$ Sonovue (Bracco, Milano, Italy) was injected in an antecubital vein in a shot bolus, washed with $10 \mathrm{ml}$ saline solution. The video recording started at the time the bolus injection ended, and before the administration of the saline solution. Five cine video-clips were recorded and stored. After 30 minutes, another $2.4 \mathrm{ml}$ of Sonovue was administrated using the same protocol, in order to examine the other hand. Particular attention was paid by the sonographer in order to avoid pressure and motion during the examination.

ROI was selected as the hypoechoic area corresponding to the synovial hypertrophy of IC and RC joints, in a dorsal, longitudinal scan. The measurement consisted of TIC parameters' calculation in "Gamma" and "Exponential" analysis, for each joint (RC and IC, respectively) (fig 1a). The same protocol was used at the follow-up visit (fig 1b).

Three parameters were analyzed: Peak intensity, AUC (both in Gamma analysis) and slope (in Exponential analysis) at baseline and at 6 months visit. In Gamma analysis, Peak intensity represents the maximum intensity of the enhancement in the time-intensity curve and is related to blood flow. AUC represents the contrast medium accumulation in the region of interest, being related to blood volume. In Exponential analysis, slope (or ascending slope) is the slope rate of ascending curve, representing the change in contrast me- dium intensity over time and quantifying the flow rate [24,30,31].

\section{Statistical analysis}

Test of normality Shapiro-Wilk was used to evaluate normality distribution. Mean \pm standard deviation (SD) was presented in the case of normally distributed data; median $\left(25^{\text {th }}-75^{\text {th }}\right.$ percentile $)$ was presented in the case of categorical variables or not normally distributed ones. When we compared initial with follow-up parameters, we performed test $t$ for paired samples or the Wilcoxon signed-rank test. For independent groups we performed the Student $t$ test for unpaired samples or the MannWhitney test. Correlation was performed by calculating Pearson coefficient of correlation for linear relationship with its significance or Spearman coefficient of correlation for categorical data or other relationship than linear.

\section{Results}

Eleven patients matched the inclusion criteria, therefore a total number of 44 joints were scanned to assess the synovial proliferation (GSUS) and vascularisation (PDUS), as well as to perform CEUS. The results showed that the joints selected as "Max" and "Min" in the case of Peak were the same joints as for AUC, while different joints were selected as "Max" and "Min" for slope. MaxPeak and maxAUC were seen at the right IC joint in 8 out of 11 patients $(72.72 \%)$; one patient $(9.09 \%)$ presented minPeak and minAUC values at this level.

No adverse reactions to the contrast agent were seen in any of the patients.

\section{Clinical assessment}

The demographics, clinical, and biological characteristics of patients at baseline and at 6 months follow up are detailed in table I. All patients were treated: at baseline, 7 (63.63\%) had Methotrexate, 3 (27.27\%) combination therapy, while at follow-up $4(36.36 \%)$ had Methotrexate alone and 5 (54.54\%) combination therapy.

\section{US analysis}

The median GSUS grades in Max Joints and Min Joints, selected according to Peak and AUC values, decreased at follow-up ( $\mathrm{p}=0.03$ and $\mathrm{p}=0.058$, respectively), while no statistical significance was found for the decrease of PDUS grades in these joint groups ( $\mathrm{p}=0.71$ and $\mathrm{p}=0.33$, respectively).

\section{CEUS analysis}

TIC parameters' values at baseline and follow-up are shown in Table II.

Correlation between TIC parameters of CEUS and the clinical parameters

Positive correlations between maximum TIC parameters and clinical features were observed, but statistical 
Table I. Demographics, clinical and biological characteristics of patients at baseline and at follow-up visit (11 patients).

\begin{tabular}{llll}
\hline & Baseline & Follow-up & p \\
\hline Parameters & & & \\
Age (years) & $55.75(15.28)$ & & \\
Male \% & 27.27 & & $0.01 / 0.003$ \\
Disease duration (months) & $8(3 ; 12)$ & $45.45 / 18.18$ & $0.01 / 0.01$ \\
Painful wrist [max\%/min\%] & $100 / 100$ & $45.45 / 45.45$ & 0.03 \\
Swollen wrist [max\%/min\%] & $100 / 100$ & $3(2 ; 6)$ & 0.01 \\
Tender joint count (0-28) & $9(5 ; 12)$ & $3(1 ; 6)$ & 0.02 \\
Swollen joint count (0-28) & $9(2 ; 13)$ & $37.27(17.37)$ & 0.004 \\
PGA (mm) & $59.54(17.58)$ & $23.63(11.42)$ & 0.03 \\
PhGA (mm) & $42.72(17.51)$ & $15(10 ; 30)$ & \\
Morning stiffness (minutes) & $30(15 ; 120)$ & & 0.14 \\
Rheumatoid factor positive \% & 81.81 & 0.16 \\
Anti CCP antibodies positive \% & 45.45 & $12(5 ; 38)$ & 0.01 \\
ESR (mm/h) & $25(15,47)$ & $0.6(0.2 ; 2.4)$ & 0.005 \\
CRP (mg/dl) & $1.02(0.6 ; 2.8)$ & $4.01(1.52)$ & 0.01 \\
DAS28 & $5.40(1.28)$ & $15.94(10.41)$ & 1 \\
SDAI & $31.93(17.56)$ & $14.63(9.31)$ & 0.92 \\
CDAI & $29.5(16.36)$ & 81.81 & \\
ACR/EULAR 2010 criteria \% & 81.81 & $15.00(8.06)$ & \\
Methotrexate & $15.45(6.50)$ & & \\
\hline Re & & \\
\hline
\end{tabular}

Results are expressed as mean (SD), median (25th;75th percentiles) or percentages (\%). PGA - patient global assessment; PhGA - physician global assessment; CCP - cyclic citrullinated peptide; ESR - erythrocyte sedimentation rate; CRP - C reactive protein; DAS28 - disease activity score 28; SDAI - symplified disease activity index; CDAI - clinical disease activity index; ACR/EULAR - American College of Rheumatology/ European League Against Rheumatism.

Table II. TIC parameters' values at baseline and at follow-up

\begin{tabular}{lllllll}
\hline & $\begin{array}{l}\text { Baseline } \\
\text { Maximum }\end{array}$ & Minimum & $\begin{array}{l}\text { Follow-up } \\
\text { Maximum }\end{array}$ & Minimum & $\begin{array}{l}\text { p } \\
\text { Maximum }\end{array}$ & Minimum \\
\hline Peak (\%) & $30(5.90)$ & $19.15(4.22)$ & $23.22(5.22)$ & $21.57(5.53)$ & 0.008 & 0.30 \\
AUC (\%s) & $1206.08(216.91)$ & $644.93(205.64)$ & $949.13(280.12)$ & $902.95(245.63)$ & 0.04 & 0.05 \\
Slope (\%/s) & $1.6(1.4 ; 2.3)$ & $0.7(0.5 ; 1)$ & $1(0.7 ; 1.2)$ & $0.8(0.5 ; 1.7)$ & 0.03 & 0.13 \\
\hline
\end{tabular}

Results are expressed in mean (standard deviation) / median (25th;75th percentiles). AUC - area under the curve

Table III. Correlations between maximum TIC parameters and clinical features

\begin{tabular}{lllll}
\hline & $\begin{array}{l}\text { Max } \boldsymbol{\Delta} \text { Peak } \\
\mathbf{r}, \mathbf{p}\end{array}$ & Max $\boldsymbol{\Delta A U C}$ & Min $\boldsymbol{\Delta \text { Peak }}$ & Min $\boldsymbol{\Delta A U C}$ \\
\hline$\Delta$ TJC & $0.05,0.88$ & $0.19,0.58$ & $-0.12,0.72$ & $-0.35,0.30$ \\
$\Delta$ SJC & $0.20,0.56$ & $0.24,0.47$ & $-0.13,0.71$ & $-0.42,0.20$ \\
$\Delta$ PGA & $0.30,0.37$ & $\mathbf{0 . 6 1 , 0 . 0 5}$ & $-0.16,0.63$ & $-0.29,0.38$ \\
$\Delta$ PhGA & $0.28,0,40$ & $0.46,0.16$ & $-0.12,0.73$ & -0.21 .0 .54 \\
$\Delta$ Morning stiffness & $0.0,0.99$ & $0.25,0.45$ & $-0.31,0.35$ & $-0.51,0.11$ \\
$\Delta$ DAS28 & $0.18,0.60$ & $0.25,0.45$ & $-0.24,0.48$ & $-0.32,0.34$ \\
$\Delta$ SDAI & $0.13,0.71$ & $0.31,0.36$ & $-0.22,0.52$ & $-0.46,0.15$ \\
$\Delta$ CDAI & $0.18,0.60$ & $0.33,0.32$ & $-0.15,0.67$ & $-0.39,0.23$ \\
\hline
\end{tabular}

$\mathrm{r}$ - Pearson's coefficient of correlation; TJC - tender joint count; SJC - swollen joint count; PGA - patient global assessment; PhGA - physician global assessment; DAS28 - disease activity score 28; SDAI - symplified disease activity index; CDAI - clinical disease activity index; Max - maximum, Min - minimum; " $\Delta$ " - delta (the difference between baseline and the follow-up visit)

significance was demonstrated only between $\triangle \mathrm{AUC}$ and $\Delta$ PGA $(\mathrm{r}=0.61, \mathrm{p}=0.05)$. Negative correlation between minimum TIC parameters and clinical features was seen, but not significant (Table III). Slope correlations with clinical parameters (data not shown) were inconclusive. For maximum $\mathrm{r}$ was between -0.14 to $0.34, \mathrm{p}=0.69$ and 0.31 , respectively; for minimum $r$ was between -0.62 to $0.42, p=0.04$ and 0.31 , respectively. 


\section{Correlation between TIC parameters of CEUS and US parameters}

Moderate correlations, without reaching statistical significance, were found between maximum $\triangle$ Peak and maximum $\triangle \mathrm{AUC}$ with maximum $\triangle \mathrm{GSUS}(\mathrm{r}=0.43$, $\mathrm{p}=0.19 ; \mathrm{r}=0.34, \mathrm{p}=0.30$, respectively) and with maximum $\triangle$ PDUS ( $r=0.44, p=0.17 ; r=0.46 ; p=0.16$, respectively).

\section{Discussions}

Two methods are described for quantifying CEUS: subjective grading according to the degree of enhancement $[21,32,33]$ and objective measurements using TIC $[32,33]$. Due to the objective quantification, TIC parameters were demonstrated to have potential added value in assessing synovial inflammation. In previous published studies, AUC was shown to correlate with PD scores [18], while AUC and slope correlated with markers of angiogenesis [34]. Our unpublished data demonstrated that the highest values of Peak, AUC and slope were seen at the right IC joint, when both wrists joints were compared in EA patients with wrist involvement.

Usually, due to the short life of the contrast agent, CEUS is performed at the most clinically affected joint $[21,23,35]$. In our study, we aimed at assessing more than one joint in one examination, and therefore we chose both wrists in patients with bilateral wrist arthritis. Subsequently we classified joint inflammation in maximum and minimum, according to the TIC parameters' values, in order to observe the evolution of extreme values.

The results of the present study indicate a significant decrease of all three maximum TIC parameters after 6 months of conventional treatment. For the minimum values, the results were not significant. In the absence of cut-off values, we do not know to which disease activity status TIC parameter values correspond at baseline and at follow-up. Different joints may become active during the evolution of RA. We observed a significant reduction in all clinical parameters used to assess the disease activity. However, mean DAS28 (4.01 in our study) at 6 months suggests a moderate clinical response, according to the EULAR response criteria, although mean $\triangle \mathrm{DAS} 28$ was higher than 1.2 [36].

In order to select the maximum TIC values, more joints need to be examined by CEUS. In our study, maxPeak and maxAUC expressed a significant decrease and therefore we think they should be focused on during follow-up. Previous published papers demonstrated a reduction of both semi-quantitative CEUS grading and slope in RA patients treated with tumor necrosis factor-alpha (TNF- $\alpha$ ) blockers. The results showed a good correlation between CEUS and magnetic resonance im- aging, suggesting that CEUS of the most clinically affected joint may be sufficient to reflect the disease activity [21].

We observed a reduction of peak and AUC in agreement with wrist pain and swelling, at follow-up, and no correlation with DAS28, SDAI or CDAI (data not shown), which may demonstrate that TIC may not reflect the disease activity as measured by the classical scores. However, the reduction of TIC parameters' values (" $\Delta$ ") may be more important. The response to therapy was assessed in previous reported works. In one study, AUC values were significantly reduced after knee intraarticular steroid injection in RA patients and correlated with clinical and PD response [19]. In another study, AUC values significantly decreased as a response to oral corticosteroids in RA patients, all patients demonstrating a reduction in AUC [23].

A trend of correlation between maximum $\triangle$ Peak and $\triangle \mathrm{AUC}$ and clinical features was observed in our study, but statistical significance was demonstrated only between $\triangle \mathrm{AUC}$ and $\triangle \mathrm{PGA}$. Negative correlations were seen between minimum $\triangle \mathrm{Peak}, \triangle \mathrm{AUC}$, and clinical parameters.

Bonifati et al [35] studied the response to TNF- $\alpha$ blockers in psoriatic arthritis using subjective grading CEUS. The results demonstrated a decrease in grade of contrast enhancement in $86.3 \%$ and that the reduction of contrast enhancement was in agreement with the evolution of clinical markers of the disease activity. However, $54 \%$ of patients positive for CEUS at baseline continued to present some degree of enhancement at follow-up, while $44 \%$ were in clinical remission [35]. Thus, it is possible that the absence of correlations between TIC parameters and the markers of clinical evolution may be a result of a continuous joint inflammation at a subclinical level.

Maximum $\triangle \mathrm{Peak}$ and $\triangle \mathrm{AUC}$ moderately correlated with maximum $\Delta$ GSUS and maximum $\triangle$ PDUS, but the results were not significant, due in part to the low number of patients. Moreover, Weidekamm et al [23] demonstrated that the decrease of AUC was observed also in cases with persistent PD. Due to the increased sensitivity of CEUS in detecting slow flow and flow in small vessels compared with PD [14,17,37], it is possible that TIC parameters detect the minimal changes in the synovial vascularisation and correlate less with PD [23].

The short enrollment period for the present study explains the low number of patients with EA and wrist involvement included. We acknowledge the reduced number of patients as one limitation of our study. Due to the low number of patients, a study for defining cutoff levels could not be performed. Further reasearch is 
needed on larger number of patients to determine cut-off levels for TIC parameters and to increase the statistical significance of the results. A test for intra- and inter-observer agreement to support the reliability data was not performed, and thus we declare an additional limitation of our study.

\section{Conclusions}

Peak and AUC for joints that had high baseline values significantly decreased following treatment with conventional synthetic drugs in EA patients with wrist arthritis. The decrease in Peak and AUC was moderately correlated with a decrease in US parameters. Importantly, Peak and AUC, but not slope, also correlated with clinical parameters. Therefore, our data support the use of Peak and AUC at the joint with the highest values of these parameters for the follow-up of EA patients.

\section{Acknowledgement}

This paper was published with financial support of the European Social Fund, Human Resources Development Operational Programme 2007-2013, POSDRU grant no. $159 / 1.5 / \mathrm{S} / 138776$

\section{Conflict of interest: none}

\section{References}

1. Albrecht K, Müller-Ladner U, Strunk J. Quantification of the synovial perfusion in rheumatoid arthritis using Doppler ultrasonography. Clin Exp Rheumatol 2007; 25: 630638.

2. Qvistgaard E, Røgind H, Torp-Pedersen S, Terslev L, Danneskiold-Samsøe B, Bliddal H. Quantitative ultrasonography in rheumatoid arthritis: evaluation of inflammation by Doppler technique. Ann Rheum Dis 2001; 60: 690-693.

3. Terslev L, Torp-Pedersen S, Savnik A, et al. Doppler ultrasound and magnetic resonance imaging of synovial inflammation of the hand in rheumatoid arthritis. Arthritis Rheum 2003; 48: 2434-2441.

4. Boesen M, Østergaard M, Cimmino MA, Kubassova O, Jensen KE, Bliddal H. MRI quantification of rheumatoid arthritis: current knowledge and future perspectives. Eur J Radiol 2009;71: 189-196.

5. Kane D, Balint PV, Sturrock RD. Ultrasonography is superior to clinical examination in the detection and localization of knee joint effusion in rheumatoid arthritis. J Rheumatol 2003; 30: 966-971.

6. Naredo E, Bonilla G, Gamero F, Uson J, Carmona L, Laffon A. Assessment of inflammatory activity in rheumatoid arthritis: a comparative study of clinical evaluation with grey scale and power Doppler ultrasonography. Ann Rheum Dis 2005; 64: 375-381.
7. Ten Cate DF, Luime JJ, Swen N, et al. Role of ultrasonography in diagnosing early rheumatoid arthritis and remission of rheumatoid arthritis - a systematic review of the literature. Arthritis Res Ther 2013; 15: R4.

8. Brown AK, Quinn MA, Karim Z, et al. Presence of significant synovitis in rheumatoid arthritis patients with diseasemodifying antirheumatic drug-induced clinical remission: evidence from an imaging study may explain structural progression. Arthritis Rheum 2006; 54: 3761-3773.

9. Saleem B, Brown AK, Keen H, et al. Should imaging be a component of rheumatoid arthritis remission criteria? A comparison between traditional and modified composite remission scores and imaging assessments. Ann Rheum Dis 2011; 70: 792-798.

10. Wakefield RJ, D'Agostino MA, Naredo E, et al. After treatto-target: can a targeted ultrasound initiative improve RA outcomes? Ann Rheum Dis 2012; 71: 799-803.

11. Filer A, de Pablo P, Allen G, et al. Utility of ultrasound joint counts in the prediction of rheumatoid arthritis in patients with very early synovitis. Ann Rheum Dis 2011; 70: 500507.

12. Salaffi F, Ciapetti A, Gasparini S, Carotti M, Filippucci E, Grassi W. A clinical prediction rule combining routine assessment and power Doppler ultrasonography for predicting progression to rheumatoid arthritis from early-onset undifferentiated arthritis. Clin Exp Rheumatol 2010; 28: 686-694.

13. Carotti M, Filippucci E, Salaffi F. Power Doppler e mezzi di contrasto nello studio della membrana sinoviale reumatoide. Reumatismo 2002; 54: 361-363.

14. De Zordo T, Mlekusch SP, Feuchtner GM, Mur E, Schirmer M, Klauser AS. Value of contrast-enhanced ultrasound in rheumatoid arthritis. Eur J Radiol 2007; 64: 222-230.

15. Klauser AS, Frauscher F, Schirmer M, et al. The value of contrast-enhanced Color Doppler ultrasound in the detection of vascularization of finger joints in patients with rheumatoid arthritis. Arthritis Rheum 2002; 46: 647-653.

16. Klauser A, Frauscher F, Schirmer M. Value of contrastenhanced power Doppler ultrasonography of the metacarpophalangeal joints on rheumatoid arthritis. Eur Radiol 2004; 14: 545-546.

17. Klauser A, Demharter J, De Marchi A, et al. Contrast enhanced gray-scale sonography in assessment of joint vascularity in rheumatoid arthritis: results from the IACUS study group. Eur Radiol 2005; 15: 2404-2410.

18. Platzgummer H, Schueller G, Grisar J, Weber M, SchuellerWeidekamm C. Quantification of synovitis in Rheumatoid Arthritis: Do we really need quantitative measurement of contrast-enhanced ultrasound? Eur J Radiol 2009; 71: 237241.

19. Salaffi F, Carotti M, Manganelli P, Filippucci E, Giuseppetti GM, Grassi W. Contrast-enhanced power Doppler sonography of knee synovitis in rheumatoid arthritis: assessment of therapeutic response. Clin Rheumatol 2004; 23: 285-290.

20. Song IH, Althoff CE, Hermann KG, et al. Contrast-enhanced ultrasound in monitoring the efficacy of a bradykinin receptor-2 antagonist in painful knee osteoarthritis 
compared to magnetic resonance imaging. Ann Rheum Dis 2009; 68: 75-83.

21. Ohrndorf S, Hensch A, Naumann L, et al. Contrast-enhanced ultrasonography is more sensitive than grayscale and power Doppler ultrasonography compared to MRI in therapy monitoring of rheumatoid arthritis patients. Ultraschall Med 2011; 32 Suppl 2: E38-E44.

22. Rednic N, Tamas MM, Rednic S. Contrast-enhanced ultrasonography in inflammatory arthritis. Med Ultrason 2011; 13: 220-227.

23. Schueller-Weidekamm C, Krestan C, Schueller G, Kapral T, Aletaha D, Kainberger F. Power Doppler sonography and pulse-inversion harmonic imaging in evaluation of rheumatoid arthritis synovitis. AJR Am J Roentgenol 2007; 188: 504-508.

24. Song IH, Althoff CE, Hermann KG, et al. Knee osteoarthritis efficacy of a new method of contrast-enhanced musculoskeletal ultrasonography in detection of synovitis in patients with knee osteoarthritis in comparison with magnetic resonance imaging. Ann Rheum Dis 2008; 67: 19-25.

25. Aletaha D, Neogi T, Silman AJ, et al. 2010 Rheumatoid arthritis classification criteria: an American College of Rheumatology/ European League Against Rheumatism collaborative initiative. Arthritis Rheum 2010 ; 62: 2569-2581.

26. Backhaus M, Burmester GR, Gerber T, et al. Guidelines for musculoskeletal ultrasound in rheumatology. Ann Rheum Dis 2001; 60: 641-649.

27. Wakefield RJ, Balint PV, Szkudlarek M, et al; OMERACT 7 Special Interest Group. Musculoskeletal ultrasound including definitions for ultrasonographic pathology. J Rheumatol 2005; 32: 2485-2487.

28. Torp-Pedersen ST, Terslev L. Settings and artefacts relevant in colour/power Doppler ultrasound in rheumatology. Ann Rheum Dis 2008; 67: 143-149.

29. Szkudlarek M, Court-Payen M, Jacobsen S, Klarlund M, Thomsen HS, Østergaard M. Interobserver agreement in ultrasonography of the finger and toe joints in rheumatoid arthritis. Arthritis Rheum 2003; 48: 955-962.

30. Dietrich CF, Averkiou MA, Correas JM, Lassau N, Leen E, Piscaglia F. An EFSUMB Introduction into Dynamic Contrast- Enhanced Ultrasound (DCE-US) for quantification of tumour perfusion. Ultraschall Med 2012; 33: 344-351.

31. Wei K, Jayaweera AR, Firoozan S, Linka A, Skyba DM, Kaul S. Quantification of myocardial blood flow with ultrasound-induced destruction of microbubbles administered as a constant venous infusion. Circulation 1998; 97: 473-483.

32. Klauser AS, Franz M, Arora R, et al. Detection of vascularity in wrist tenosynovitis: power doppler ultrasound compared with contrast-enhanced grey-scale ultrasound. Arthritis Res Ther 2010; 12: R209.

33. Klauser AS, Franz M, Bellmann Weiler R, et al. Contrastenhanced ultrasonography for the detection of joint vascularity in arthritis-subjective grading versus computer-aided objective quantification. Ultraschall Med 2011; 32 Suppl 2: E31-E37.

34. Jiang Y, Qiu L, Zhang L, Luo Y, Lin L, Wen X. Noninvasive quantitative assessment of synovial pannus angio- genesis by contrast-enhanced gray-scale sonography in antigeninduced arthritis in rabbits. Acad Radiol 2011; 18: 359-368.

35. Bonifati C, Elia F, Graceffa D, et al. Clinical and ContrastEnhanced Ultrasound Echography Outcomes in Psoriatic Arthritis Patients after One Year of Continuous Therapy with Anti-TNF Drugs. ISRN Dermatol 2014; 2014: 932721.

36. Fransen J, van Riel PL. The Disease Activity Score and the EULAR response criteria. Clin Exp Rheumatol 2005; 23 (Suppl 39): S93-S99.

37. Terslev L, Torp-Pedersen S, Bang N, Koenig MJ, Nielsen $\mathrm{MB}$, Bliddal H. Doppler ultrasound findings in healthy wrists and finger joints before and after use of two different contrast agents. Ann Rheum Dis 2005; 64: 824-827. 